\title{
The detection of gastric cancer cells in intraoperative peritoneal lavage using the RT-LAMP method
}

Akira Yoneda, Ken Taniguchi, Yasuhiro Torashima, Seiya Susumu, Kengo Kanetaka,

Tamotsu Kuroki, Susumu Eguchi

Department of Surgery, Nagasaki University Graduate School of Biomedical Sciences,

Nagasaki, Japan

Reprint requests: Akira Yoneda, Department of Surgery, Nagasaki University Graduate

School of Biomedical Sciences, 1-7-1 Sakamoto, Nagasaki 852-8501, Japan,

Tel.: +81-95-819-7316; Fax: +81-95-819-7319; E-mail: dm06034e@cc.nagasaki-u.ac.jp

Running title: The detection of gastric cancer cells using the RT-LAMP 


\section{Abstract}

\section{Introduction}

To detect a small number of malignant cells, we used a highly sensitive detection system that measures the expression levels of cytokeratin 19-mRNA by reverse-transcription loop-mediated isothermal amplification (RT-LAMP).

\section{Materials and methods}

We evaluated the clinical relevance of our novel diagnostic method with an RT-LAMP assay using cytokeratin 19 as a target gene for the detection of free cancer cells in peritoneal lavage, and assessed the clinical significance of the molecular diagnosis by survival analysis and frequency of recurrence, with a median follow-up period of 39 months. We observed 52 patients with gastric cancer who underwent gastrectomy, bypass operation and exploratory laparotomy.

\section{Results}

Those 52 patients, who were subjected to both RT-LAMP and cytological examination, were divided into the following three groups: (1) patients who were positive by cytology and RT-LAMP (CY+/LAMP+) $(n=9),(2)$ patients positive by LAMP and negative by cytology (CY-/LAMP+) $(n=12)$, and (3) patients negative by 
both cytology and LAMP (CY-/LAMP-) $(\mathrm{n}=31)$. All patients with simultaneous peritoneal dissemination and positive cytology were positive on RT-LAMP. The results of RT-LAMP were statistically significant for recurrence by univariate analysis $(\mathrm{P}<0.005)$. Cytology-positive cases had a very poor prognosis, and RT-LAMP-positive cases had a worse prognosis than RT-LAMP-negative cases.

\section{Conclusion}

Our findings suggest that CK-19 RT-LAMP would be useful as an intraoperative diagnostic modality in order to detect patients with a high risk of recurrence even after clinically curative surgery, who thus require proper adjuvant therapy.

Key words: gastric cancer, RT-LAMP, peritoneal lavage 


\section{Introduction}

Peritoneal carcinomatosis is the most frequent pattern of recurrence in patients with gastric cancer ${ }^{1-2)}$. The prognosis of patients with advanced gastric cancer invading the gastric serosa is very poor even after curative resection, mainly because of the high incidence of peritoneal recurrence ${ }^{3)}$. Recurrence with this pattern is most likely caused by the presence of free cancer cells in the abdominal cavity exfoliated from the serosal surfaces of the primary gastric tumor ${ }^{4)}$. Therefore, detection of such micrometastatic cells in the peritoneal cavity is likely to be a useful tool in the selection of intra/post-operative chemotherapy as well as for predicting the outcome of such therapy in these cases ${ }^{5}$. In this regard, cytologic examination of lavage fluid obtained at the time of surgery is a conventional method for detecting free cancer cells in peritoneal space. However, the sensitivity of this assay has been reported to be relatively low, ranging from $19 \%$ to $30 \%$ in gastric cancer invading the serosa ${ }^{6-9)}$. As a result, some patients with negative cytology have nevertheless developed peritoneal recurrence. Therefore, there is an urgent need for more sensitive methods to detect micrometastasis in the peritoneal cavity. To detect small numbers of malignant cells among the cytologically negative cases, we developed a highly sensitive detection system that measures the expression levels of cytokeratin (CK) 19-mRNA by reverse-transcription 
loop-mediated isothermal amplification (RT-LAMP). The RT-LAMP method is a new method of gene amplification, the efficacy of which has been reported ${ }^{10-11)}$. The reaction is accelerated by the use of two additional loop primers (called Loops F and B) ${ }^{11)}$. The LAMP method can be conducted simultaneously with reverse transcription from mRNA (reverse transcriptase-LAMP: RT-LAMP) ${ }^{10-14)}$. There are several practical advantages to the RT-LAMP technique: it requires only simple reaction procedures, the compact and inexpensive incubator/turbidimeter equipment costs less than $\$ 5,000$, and less than $1 \mathrm{hr}$ is needed to obtain the final results ${ }^{11-15)}$. Application of the LAMP technique has been reported for breast cancer and lung cancer ${ }^{16-18)}$. This technique might be one of the most promising candidates for analyzing the genetic features of samples obtained during surgery.

CK proteins of the intermediate filaments of epithelial cells have been used as specific markers for tumor cells of epithelial origin ${ }^{19-20)}$. In the present study, we evaluated the clinical relevance of a new diagnostic method employing an RT-LAMP assay with CK19 as the target gene for the detection of free cancer cells in the peritoneal lavage, and assessed the clinical significance of the molecular diagnosis by survival analysis and frequency of recurrence. 


\section{Materials and methods}

\section{Cell lines}

A sensitivity assay for detecting a gastric cancer cell line was performed. The human gastric cancer cell line MKN-45, obtained from the Riken Cell Bank (Institute of Physical and Chemical Research, Saitama, Japan) was incubated in RPMI-1640 medium containing $10 \%$ fetal calf serum (Invitrogen, Carlsbad, CA, USA) at $37^{\circ} \mathrm{C}$ in $5 \% \mathrm{CO}_{2}$.

\section{Patients}

Between May 2007 and November 2008, we observed 52 patients (35 males and 17 females; mean age, $67.5 \pm 2.8$ years) with gastric cancer who underwent gastrectomy $(n=45)$, bypass operation $(n=2)$ and exploratory laparotomy $(n=7)$ for histologically proven gastric cancer at the Department of Surgery, Nagasaki University. Written informed consent for participation in this study was obtained from all patients. All were followed up for a median of 39 months (range, 6-51 months) or until death. The primary tumor was resected in 45 of the 52 patients ( 5 patients had peritoneal dissemination, but underwent resection of their primary tumor because of the stenosis and bleeding caused by primary tumor as a pariative treatment) but was unresectable in 7 patients due to peritoneal dissemination and positive cytology. These 7 patients underwent a bypass 
operation or exploratory laparotomy. The resected specimens were histologically examined by hematoxilin and eosin staining according to the general rules of the Japanese Classification of Gastric Carcinoma ${ }^{21)}$. Clinicopathological features of the patients are shown in Table 1.

\section{RT-LAMP reaction}

LAMP primers targeting the CK19 cDNA were designed based on a past report ${ }^{22)}$ (Fig. 1). To quantify and prove the integrity of isolated RNA, we also performed RT-LAMP for $\beta$-actin.

The RT-LAMP method was carried out on $25 \mu \mathrm{l}$ of the total reaction mixture with a Loopamp RNA amplification kit (Eiken Chemical Co., Tokyo, Japan) containing 40 pmol each of the forward (FIP) and backward inner primers (BIP), 5 pmol each of the outer primers F3 and B3, 20 pmol each of the loop primers loop F and loop B, 35 pmol dNTPs, 20- $\mu$ l Betamine, 0.5 $\mu \mathrm{M}$ Tris-HCL (pH 8.8), 0.25 $\mu \mathrm{M}$ KCL, $0.25 \mu \mathrm{M}$ (NH4)SO4, $0.2 \mu \mathrm{M}$ MgSO4, 0.2\% Tween20, $1.0 \mu \mathrm{l}$ Enzyme Mix (Bst DNA polymerase and avian myeloblastosis virus (AMV) reverse transcriptase) and $5 \mu \mathrm{l}$ RA at a constant temperature of $63.5^{\circ} \mathrm{C}$ for $60 \mathrm{~min}$. Temperature control for the LAMP reaction and turbidity measurement was achieved using a turbidimeter (LA-200; Teramecs Co., Kyoto, Japan) especially developed for DNA analysis by LAMP reaction. 


\section{Sensitivity evaluation of RT-LAMP on CK19 mRNA detection}

A fundamental experiment was performed to determine the sensitivity of the

RT-LAMP method for detecting gastric cancer cells in peripheral blood mononuclear

cells (PBMCs). Peripheral venous blood was obtained from healthy volunteers. Gastric cancer MKN-45 cells were serially diluted from $1 \times 10^{6}$ cells to one cell per $1 \times 10^{7}$ PBMCs. The mRNA was extracted from each cell fraction, and RT-LAMP for CK19 mRNA was performed.

\section{Preparation of peritoneal floating cells}

At the beginning of the operation, before the manipulation of the tumor, $400 \mathrm{ml}$ of physiological saline was introduced into the upper abdominal cavity and recovered after being gently stirred. Part of the peritoneal lavage fluid was subjected to conventional cytology after standard Papanicolaou staining, and the remaining fluid was immediately centrifuged at $2000 \mathrm{rpm}$ for $10 \mathrm{~min}$. The pellets of lavaged fluid were rinsed with phosphate-buffered saline (PBS), dissolved in RNAlater ${ }^{\mathrm{TM}}$ solution (Ambion, Austin, TX) and stored at $-80^{\circ} \mathrm{C}$ until use.

\section{mRNA extraction}

mRNA was extracted using the Dynabeads mRNA DIRECT kit (Veritas, Tokyo) according to the manufacturer's instructions. Briefly, collected cells were lysed by the 
Lysis/binding buffer, and the released mRNA, and its poly (A) residue were hybridized with oligo (dT) conjugated with Dynabeads; then this complex was immobilized onto the surface of magnetic beads. Contaminating components were washed away by repeated steps of separation and resuspension in washing buffer. Finally, the purified mRNA was eluted from the particles.

\section{RT-LAMP on mRNA derived from peritoneal lavage}

$25 \mu \mathrm{l}$ of reaction mixture was applied to each reaction. Positive control primers ( $\beta$-actin) and a negative control mixture (PBMC-derived sample) were employed for all reactions.

\section{Statistical analysis}

All statistical analyses were performed using StatView 5.0 software (SAS Institute, Cary, NC, USA). The chi-squared test was used to evaluate the correlation between positive results and clinicopathological factors, and univariate analysis was used to evaluate the results of RT-LAMP and recurrence. Survival curves were calculated using the Kaplan-Meier method. Survival curves were compared using the log rank test. $\mathrm{P}<0.05$ was considered statistically significant. 


\section{Results}

Evaluation of the sensitivity of RT-LAMP for identifying gastric carcinoma cell line As few as $10^{0}$ MKN45 cells in $10^{7}$ normal PBMCs were detectable with the RT-LAMP procedures targeting CK19 mRNA using extracted mRNA of cell mixtures lysate (Fig. 2).

Detection of cancer cells in intraoperative peritoneal lavage

The mRNA of $\beta$-actin, a housekeeping gene, was detected in all of the present samples. RT-LAMP reaction diagnosis significantly correlated with lymph node metastasis, depth of invasion, lymphatic invasion and vessel invasion (Table 2). The total of 52 patients who were subjected to both RT-LAMP and cytological examination were divided into the following three groups: (1) patients who were positive by cytology and RT-LAMP (CY+/LAMP+) (n=9), (2) patients positive by LAMP and negative by cytology (CY-/LAMP+) ( $\mathrm{n}=12)$, and (3) patients negative by both cytology and LAMP (CY-/LAMP-) (n=31). The stage of each groups were showed on Table2. There were no patients negative by LAMP and positive by cytology; all patients with simultaneous peritoneal dissemination and positive cytology were positive on RT-LAMP. Simultaneous peritoneal dissemination at surgery or staging laparoscopy was detected in 12 patients (9 in the group CY+/LAMP+ and 3 in the group CY-/LAMP+). No patients 
with negative RT-LAMP and negative CY status had recurrence after surgery. The results of RT-LAMP were statistically significant for recurrence by univariate analysis $(\mathrm{P}<0.005)$. Fig. 3 shows the overall survival (Fig. 3A), recurrence-free survival (Fig. 3B) and peritoneal recurrence-free survival (Fig. 3C) curves for the three patient groups subjected to both RT-LAMP and cytological examination. Fig. 3 shows that cytology-positive cases had a very poor prognosis and that cases with negative cytology but positive RT-LAMP had a worse prognosis than that of cases who were RT-LAMP-negative. 


\section{Discussion}

Although the standard surgical lymphadenectomy for gastric cancer has been established and applied, patients with advanced stages of this cancer continue to face a poor prognosis. A previous study suggested that the presence of peritoneal free cancer cells was responsible for the formation of micrometastasis and subsequent extensive dissemination ${ }^{23-24)}$. Cytological examination aimed at the detection of these cells, therefore, has been generally accepted as the golden criterion for the prediction of peritoneal recurrence, and this procedure has been incorporated in the Japanese staging system for gastric cancer ${ }^{21)}$. However, peritoneal recurrence sometimes occurs in patients with negative cytology, which indicates the lack of sensitivity of conventional cytological examination for the prediction of peritoneal recurrence. We believe that it is difficult to confirm peritoneal metastasis from cytology because of its low sensitivity. Jung et al. reported that even in patients with clinically diagnosed carcinomatosis with ascites, only $54 \%$ of patients were positive for cytology. ${ }^{25)}$

Thus, a more sensitive assay for the detection of peritoneal micrometastasis is required.

New, simple and rapid molecular techniques for the detection of target genes have been developed in recent years, and one of these is the LAMP reaction. This reaction is a novel approach to the DNA amplification of target sequences, providing high 
sensitivity, specificity and rapidity under isothermal conditions. The LAMP reaction relies on auto-cycling strand displacement DNA synthesis that is performed with a DNA polymerase with high strand displacement activity and four specific primers recognizing six independent sequences. Specifically, it synthesizes a large amount of amplification products, comprising a mixture of stem-loop DNA and cauliflower-like structures with multiple loops (except for the loops that are hybridized by the inner primer) and prime strand displacement DNA. When the target DNA is amplified by LAMP reaction, a white precipitate derived from magnesium pyrophosphate (a byproduct of LAMP reaction) is observed ${ }^{10)}$; thus, the LAMP method does not require special reagents or electrophoresis to detect the amplified DNA. Amplification of the targeted gene is detectable in real-time fashion by an increase of the turbidity of the solution.

CK19 has been shown to be widely expressed by cancer cells of epithelial origin but not by lymphoid or hematopoietic cells ${ }^{26}$. Among some 20 different isotypes, CK19 and CK20 are expressed more selectively by mucosal epithelial cells than are the others ${ }^{27)}$. It has been reported that CK19 is superior to CK20 in detecting circulating cancer cells using RT-PCR in peripheral blood from patients with gastric cancer ${ }^{28)}$. Thus, this molecule may be a suitable general marker of micrometastasis in peritoneal lavage of patients with gastric cancer. LAMP primers were generated to detect the 
CK19 sequence, and the performance of the RT-LAMP reaction to detect gastric cancer cells was tested. Only cells of epithelial origin were detected by this technique, with sensitivity to concentrations as low as one cell per $10^{7}$ normal PBMCs. CK expression was observed in the RT-LAMP reaction, presumably due to its high specificity, which requires the recognition of six independent sequences within the target molecule ${ }^{22)}$. The development of a rapid technique for detecting cancer cells in peritoneal lavage of patients with gastric cancer using the RT-LAMP reaction is herein described. Among the patients with negative cytology, those with a positive RT-LAMP reaction had a poorer prognosis than that of those with negative RT-LAMP reaction results. In three patients, laparotomy was performed preceding the staging laparoscopy to verify negative cytology and the lack of obvious peritoneal dissemination. However, peritoneal dissemination was found after open exploration, and further surgery was cancelled. For all of these patients, the results of the RT-LAMP reaction were positive. As these results indicated, even when cancer cells are not found by cytology, the RT-LAMP method could be used to determine the necessity of surgery. Our results demonstrated significant correlations between the CK19 findings and the depth of cancer invasion, the presence and extent of lymph node metastasis, and the vessel and lymphatic invasion. In this sense, CK19 expressed by peritoneal free cancer cells could be a candidate 
molecular marker indicating high invasive potential and aggressive behavior in gastric cancers. It is remarkable that the results of the RT-LAMP method correlated not only with the peritoneal recurrences but also with other fashions without tumor exposure to the serosa. Minor diffusion of tumor cells might occur during the migration through the lymphatic vessels ${ }^{29-31)}$, and further evaluation would be needed around the origin. The benefit of adjuvant chemotherapies in solid tumors is known to be related to the amount of remnant tumor burden. Therefore, patients with positive RT-LAMP without macroscopic peritoneal dissemination seem good candidates for a cure through appropriate adjuvant therapy. Kodera et al. reported that postoperative S-1 monotherapy could make no difference in survival between patients with visible peritoneal deposits and patients with only positive cytology ${ }^{32)}$. To improve the prognosis, it is crucial to identify high-risk patients at a much earlier phase of peritoneal dissemination. As molecular approaches such as RT-LAMP and RT-PCR analysis have the potential to do just that, adjuvant therapy could eliminate remnant cancer cells detected only by molecular diagnosis. Limitations include a small sample size. The RT-LAMP technique could be performed as an alternative to an intraoperative cytologic examination. Because of its high sensitivity and rapidity, this method could provide an opportunity to perform reliable tailor-made surgery for gastric cancers as a 
common procedure in general hospitals. However, the present study has some limitations. First, this study included a too small sample size to assess whether RT-LAMP positivity could be independent prognostic biomarker in cytology negative patients. Second, this molecular-based method is known to be concerned with a high false positive rate. Kodera et al reported that when the cutoff value was set at 0.1 for CEA mRNA, the false positive rate of CEA RT-PCR exceeded $10 \%$. ${ }^{33)}$ To confirm the efficacy of the RT-LAMP method, additional efforts are necessary to assess the outcome of the treatment of patients with RT-LAMP-positive peritoneal lavage findings in larger sample size.

\section{Conclusion}

Our findings suggest that CK-19 RT-LAMP would be useful as an intraoperative diagnostic modality in order to detect potential high-risk patients who may develop recurrence even after clinically curative surgery, and to guide choices about proper adjuvant therapy. 


\section{Figure legends}

Figure 1: Primer design for detection of CK19 mRNA by RT-LAMP.

Figure 2: Sensitivity analysis of the RT-LAMP method. As few as $10^{0} \mathrm{MKN} 45$ cells in $10^{7}$ normal PBMCs were detectable with the RT-LAMP procedures targeting CK19 mRNA using extracted mRNA of the cell mixture lysate.

Figure 3A: Overall survival curves of patients stratified according to results of cytology and RT-LAMP. Significant differences between the CY-LAMP- and CY-LAMP+ groups were found $(\mathrm{P}<0.05)$.

Figure 3B: Recurrence-free survival curves of patients stratified according to the results of cytology and RT-LAMP. Significant differences between the CY-LAMPand CY-LAMP+ groups were found $(\mathrm{P}<0.05)$.

Figure 3C: Peritoneal recurrence-free survival curves of patients stratified according to results of cytology and RT-LAMP. Significant differences between the CY-LAMP- and CY-LAMP+ groups were found $(\mathrm{P}<0.05)$. 


\section{References}

1. Yoo CH, Noh SH, Shin DW, et al. Recurrence following curative resection for gastric carcinoma. Br J Surg 2000;87: 236-242.

2. Maehara Y, Hasuda S, Koga T, et al. Postoperative outcome and sites of recurrence in patients following curative resection of gastric cancer. Br J Surg 2000;87: 353-357.

3. Baba H, Korenaga D, Okamura T, et al. Prognostic factors in gastric cancer with serosal invasion. Univariate and multivariate analyses. Arch Surg 1989;124: 1061-1064.

4. Koga S, Kaibara N, litsuka Y, et al. Prognostic significance of intraperitoneal free cancer cells in gastric cancer patients. J Cancer Res Clin Oncol 1984;108: 236-238.

5. Bonenkamp JJ, Songun I, Hermans J, et al. Prognostic value of positive cytology findings from abdominal washings in patients with gastric cancer. Br J Surg 1996;83: 672-674.

6. Ikeguchi M, Oka A, Tsujitani S, et al. Relationship between area of serosal invasion and intraperitoneal free cancer cells in patients with gastric cancer. Anticancer Res 1994;14: 2131-2134. 
7. Burke EC, Karpeh MS, Jr., Conlon KC, et al. Peritoneal lavage cytology in gastric cancer: an independent predictor of outcome. Ann Surg Oncol 1998;5: 411-415.

8. Kodera Y, Yamamura Y, Shimizu Y, et al. Peritoneal washing cytology: prognostic value of positive findings in patients with gastric carcinoma undergoing a potentially curative resection. J Surg Oncol 1999;72: 60-64; discussion 64-65.

9. Suzuki T, Ochiai T, Hayashi H, et al. Importance of positive peritoneal lavage cytology findings in the stage grouping of gastric cancer. Surg Today 1999;29: 111-115.

10. Notomi T, Okayama H, Masubuchi H, et al. Loop-mediated isothermal amplification of DNA. Nucleic Acids Res 2000;28: E63.

11. Nagamine K, Hase T, Notomi T Accelerated reaction by loop-mediated isothermal amplification using loop primers. Mol Cell Probes 2002;16: 223-229.

12. Hirayama H, Kageyama S, Moriyasu S, et al. Rapid sexing of bovine preimplantation embryos using loop-mediated isothermal amplification. Theriogenology 2004;62: 887-896.

13. Parida M, Posadas G, Inoue S, et al. Real-time reverse transcription 
loop-mediated isothermal amplification for rapid detection of West Nile virus. J

Clin Microbiol 2004;42: 257-263.

14. Ushio M, Yui I, Yoshida N, et al. Detection of respiratory syncytial virus genome by subgroups-A, B specific reverse transcription loop-mediated isothermal amplification (RT-LAMP). J Med Virol 2005;77: 121-127.

15. Mori Y, Nagamine K, Tomita N, et al. Detection of loop-mediated isothermal amplification reaction by turbidity derived from magnesium pyrophosphate formation. Biochem Biophys Res Commun 2001;289: 150-154.

16. Tsujimoto M, Nakabayashi K, Yoshidome K, et al. One-step nucleic acid amplification for intraoperative detection of lymph node metastasis in breast cancer patients. Clin Cancer Res 2007;13: 4807-4816.

17. Maeda J, Inoue M, Nakabayashi K, et al. Rapid diagnosis of lymph node metastasis in lung cancer with loop-mediated isothermal amplification assay using carcinoembryonic antigen-mRNA. Lung Cancer 2009;65: 324-327.

18. Tamaki Y, Akiyama F, Iwase T, et al. Molecular detection of lymph node metastases in breast cancer patients: results of a multicenter trial using the one-step nucleic acid amplification assay. Clin Cancer Res 2009;15: 2879-2884.

19. Moll R, Franke WW, Schiller DL, et al. The catalog of human cytokeratins: 
patterns of expression in normal epithelia, tumors and cultured cells. Cell 1982;31: 11-24.

20. Osborn M, van Lessen G, Weber K, et al. Differential diagnosis of gastrointestinal carcinomas by using monoclonal antibodies specific for individual keratin polypeptides. Lab Invest 1986;55: 497-504.

21. Japanese classification of gastric carcinoma: 3rd English edition. Gastric Cancer 14: $101-112$.

22. Horibe D, Ochiai T, Shimada H, et al. Rapid detection of metastasis of gastric cancer using reverse transcription loop-mediated isothermal amplification. Int J Cancer 2007;120: 1063-1069.

23. Wong SC, Yu H, So JB Detection of telomerase activity in gastric lavage fluid: a novel method to detect gastric cancer. J Surg Res 2006;131: 252-255.

24. Ishigami S, Sakamoto A, Uenosono Y, et al. Carcinoembryonic antigen messenger RNA expression in blood can predict relapse in gastric cancer. J Surg Res 2008;148: 205-209.

25. Jung M, Jeung HC, Lee SS, et al. The clinical significance of ascitic fluid CEA in advanced gastric cancer with ascites. J Cancer Res Clin Oncol 136: 517-526.

26. Ruud P, Fodstad O, Hovig E Identification of a novel cytokeratin 19 pseudogene 
that may interfere with reverse transcriptase-polymerase chain reaction assays used to detect micrometastatic tumor cells. Int J Cancer 1999;80: 119-125.

27. Moll R, Lowe A, Laufer J, et al. Cytokeratin 20 in human carcinomas. A new histodiagnostic marker detected by monoclonal antibodies. Am J Pathol 1992;140: 427-447.

28. Majima T, Ichikura T, Takayama E, et al. Detecting circulating cancer cells using reverse transcriptase-polymerase chain reaction for cytokeratin mRNA in peripheral blood from patients with gastric cancer. Jpn J Clin Oncol 2000;30: 499-503.

29. Yamagata K, Kumagai K Experimental study of lymphogenous peritoneal cancer dissemination: migration of fluorescent-labelled tumor cells in a rat model of mesenteric lymph vessel obstruction. J Exp Clin Cancer Res 2000;19: 211-217.

30. Sleeman JP, Thiele W Tumor metastasis and the lymphatic vasculature. Int $\mathrm{J}$ Cancer 2009;125: 2747-2756.

31. Hirakawa S From tumor lymphangiogenesis to lymphvascular niche. Cancer Sci 2009;100: 983-989.

32. Kodera Y, Ito S, Mochizuki Y, et al. A phase II study of radical surgery followed 
by postoperative chemotherapy with $\mathrm{S}-1$ for gastric carcinoma with free cancer cells in the peritoneal cavity (CCOG0301 study). Eur J Surg Oncol 2009;35: 1158-1163.

33. Kodera Y, Nakanishi H, Ito S, Mochizuki Y, Ohashi N, Yamamura Y, et al. Prognostic significance of intraperitoneal cancer cells in gastric carcinoma: analysis of real time reverse transcriptase-polymerase chain reaction after 5 years of followup. J Am Coll Surg 2006;202:231-6. 
Figure 1

F3: CCTCCTACCTGGCAAGGT

B3: ATGCGCAGAGCCTGTC

FIP(F1c+F2):TAGTGGCTGTAGTCGCGG

GAAACGGCGAGCTAGAGGAGAA

BIP (B1C+B2):CGGGACAAGATTCTTGGTGCCAAAGT

CATCTGCAGCCAGACG

loopF : AGGCCCCTGCTTCTGGTAC

loopB: CCTGCAGATCGACAATGCC 
Figure 3A

Overall survival of patients

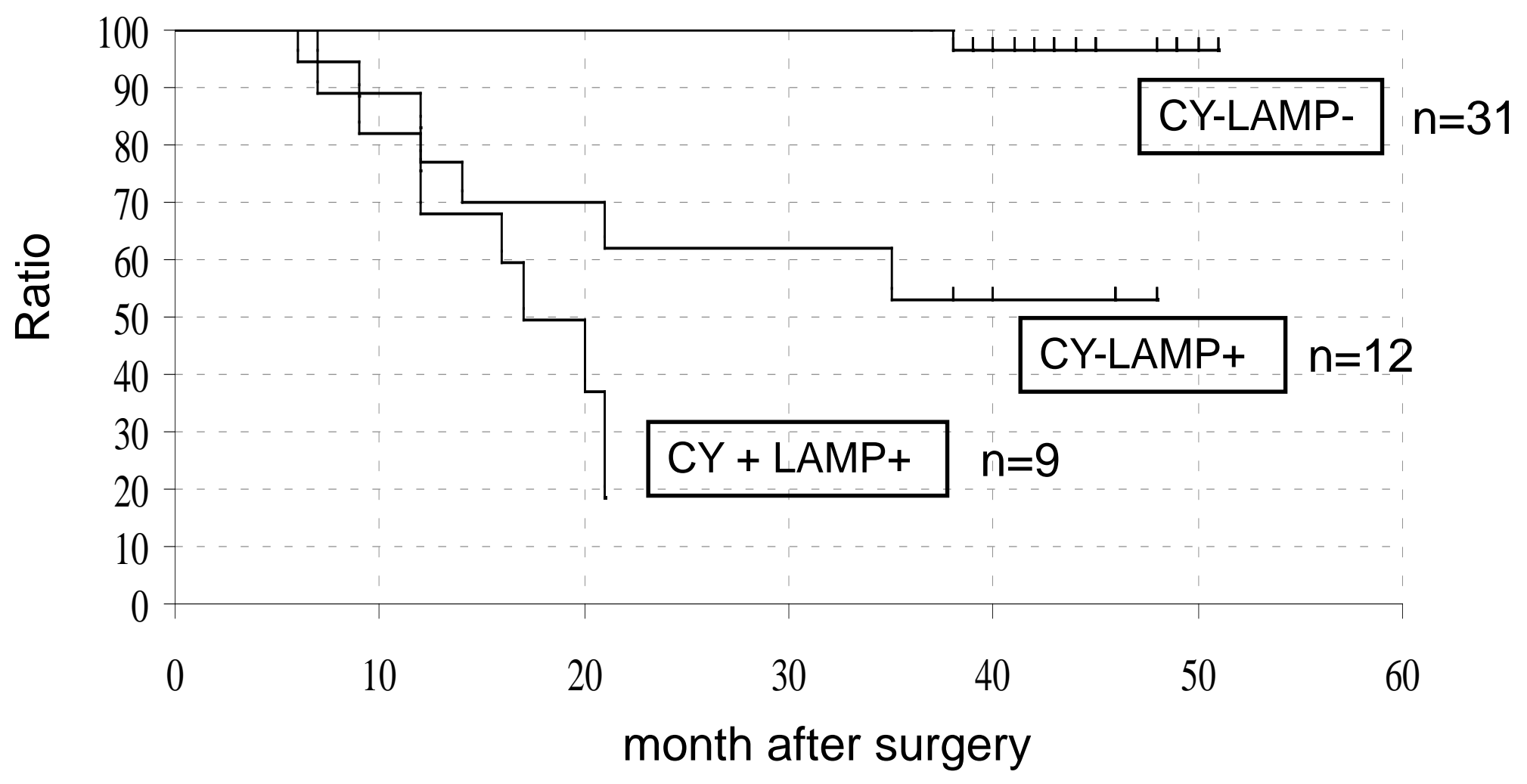


Figure 3B

Recurrence-free survival of patients

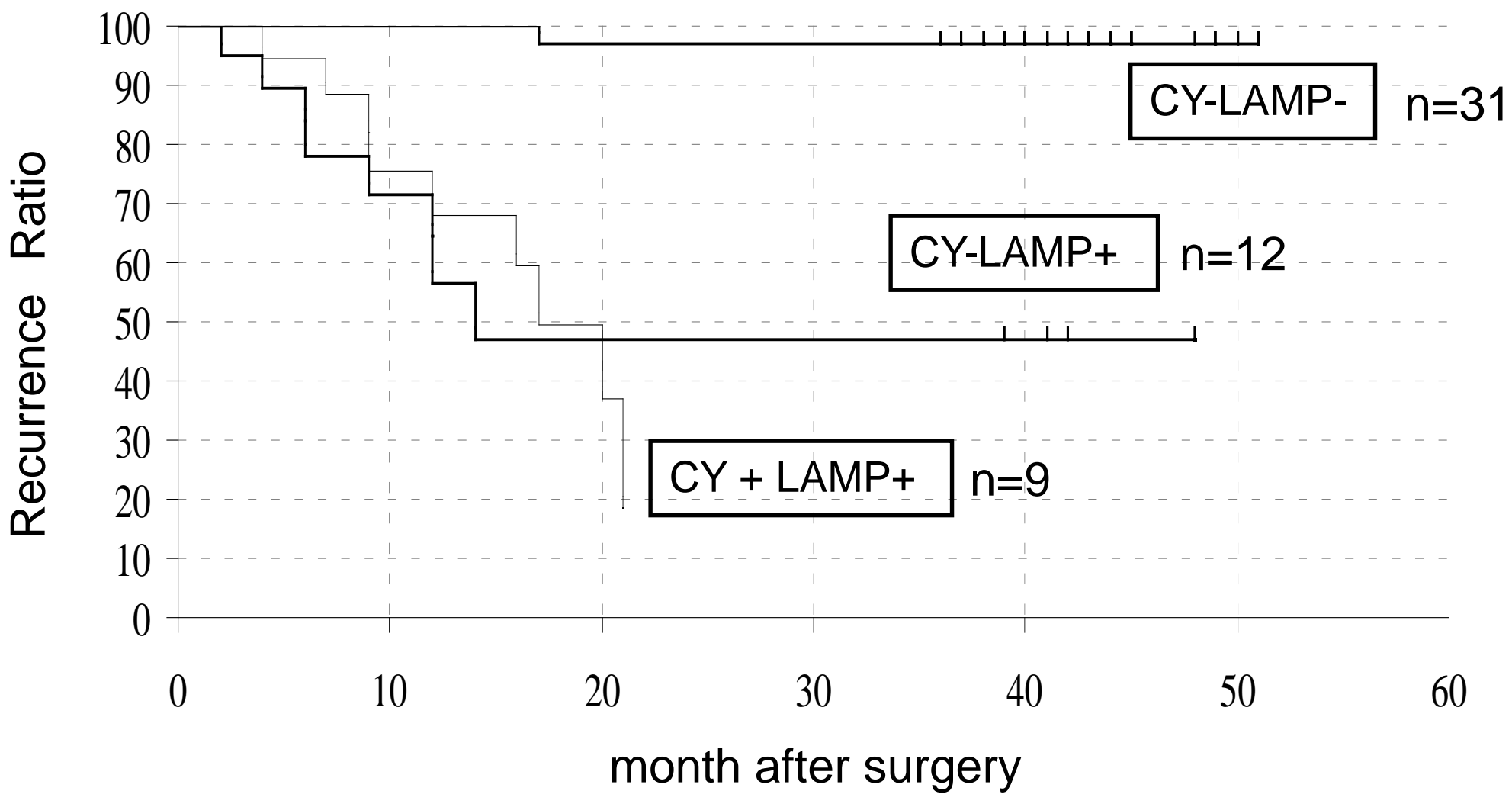


Figure 3C

Peritoneal recurrence-free survival of patients

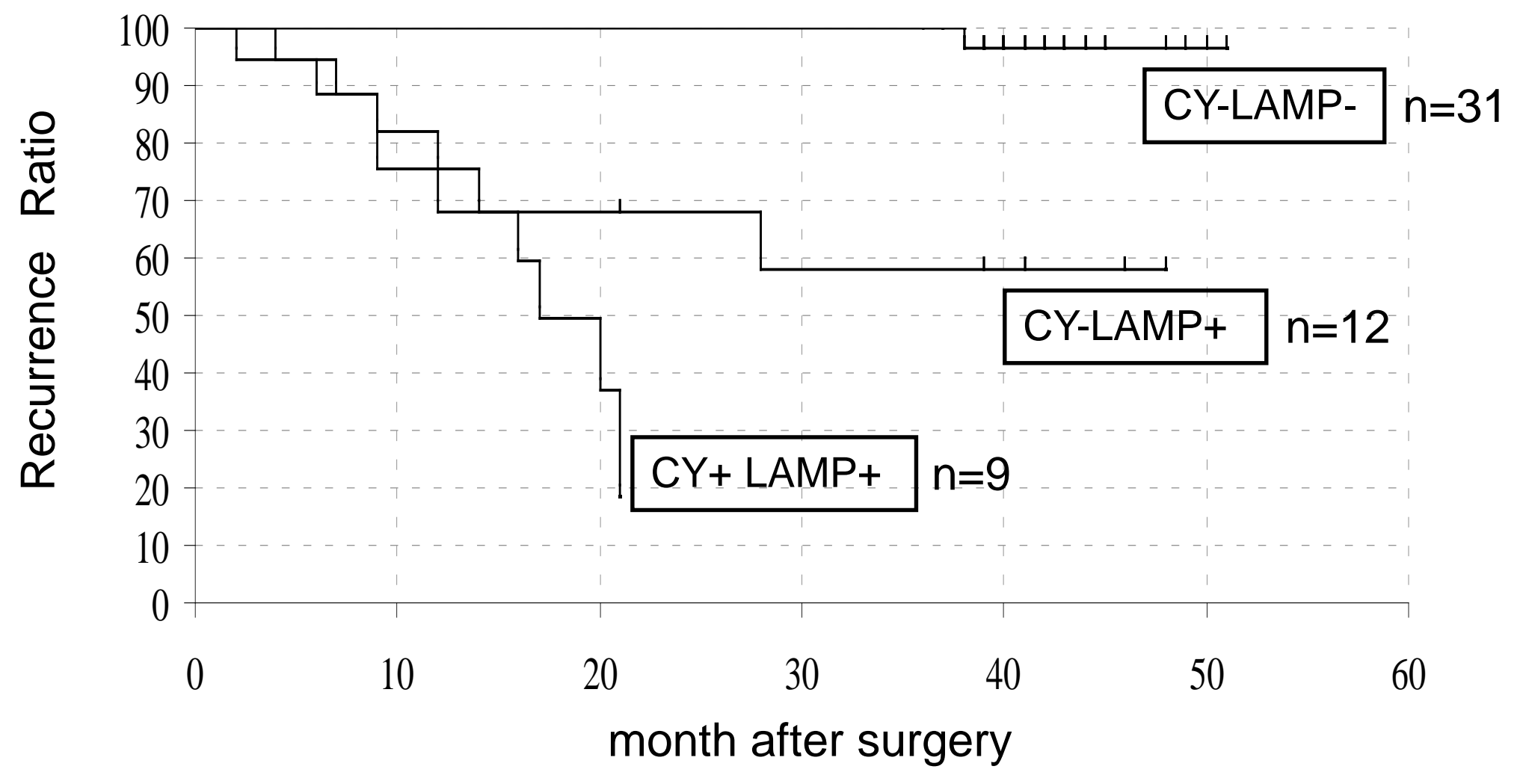


Table 1: Clinicopathological factors were determined according to the Japanese Classification of Gastric Carcinoma

\begin{tabular}{|c|c|c|}
\hline & & No of patients \\
\hline \multicolumn{2}{|l|}{ Total cases } & 52 \\
\hline \multicolumn{2}{|l|}{ Age(yr) } & $67.5 \pm 2.8$ \\
\hline \multicolumn{2}{|l|}{ Sex Male/Female } & $35 / 17$ \\
\hline \multirow[t]{5}{*}{ Depth of tumor invasion } & M & 11 \\
\hline & SM & 13 \\
\hline & MP & 6 \\
\hline & SS & 9 \\
\hline & SE, SI & 13 \\
\hline \multirow[t]{4}{*}{ Lymph node metastasis } & N0 & 22 \\
\hline & N1 & 13 \\
\hline & N2 & 13 \\
\hline & N3 & 4 \\
\hline \multirow[t]{2}{*}{ Peritoneal metastasis } & Absent & 40 \\
\hline & Present & 12 \\
\hline \multirow[t]{8}{*}{ Cytology } & Negative & 43 \\
\hline & Positive & 9 \\
\hline & & \\
\hline & Stage IA & 18 \\
\hline & Stage IB & 7 \\
\hline & Stage II & 8 \\
\hline & Stage III & 4 \\
\hline & Stage IV & 15 \\
\hline \multicolumn{3}{|c|}{ M, mucosa; SM, submucosa; MP, muscularis propria; SS, subserosa; } \\
\hline
\end{tabular}


Table 2: Correlation between RT-LAMP diagnosis of peritoneal lavage and clinicopathological parameters and the stage of each gourps.

\begin{tabular}{|c|c|c|c|c|}
\hline & & \multicolumn{3}{|c|}{ Expresson of CK19 } \\
\hline & & \multicolumn{3}{|c|}{ mRNA (RT-LAMP) } \\
\hline & & Positive & Negative & P-value \\
\hline No of patients & & 21 & 31 & \\
\hline \multirow[t]{2}{*}{ Sex } & Male & 14 & 21 & N.S. \\
\hline & Female & 7 & 10 & \\
\hline \multirow[t]{3}{*}{ Age (yr) } & & 62.45 & 67.8 & N.S. \\
\hline & M & 0 & 11 & \\
\hline & SM & 0 & 13 & \\
\hline \multirow[t]{3}{*}{ Depth of tumor invasion } & MP & 3 & 3 & 0.00001 \\
\hline & SS & 6 & 3 & \\
\hline & SE,SI & 12 & 1 & \\
\hline \multirow[t]{2}{*}{ Lymph node metastasis } & - & 3 & 23 & 0.00002 \\
\hline & + & 18 & 8 & \\
\hline \multirow[t]{2}{*}{ Lymphatic invasion } & - & 1 & 16 & 0.0091 \\
\hline & + & 11 & 15 & \\
\hline \multirow[t]{2}{*}{ Vessel invasion } & - & 1 & 18 & 0.0033 \\
\hline & + & 11 & 13 & \\
\hline
\end{tabular}


M, mucosa; SM, submucosa; MP, muscularis propria; SS, subserosa;

SE, serosa exposed; SI, serosa infiltrating.

$\mathrm{CY}+/ \mathrm{LAMP}+(\mathrm{n}=9) \quad$ stage IV; $\mathrm{n}=9$

CY-/LAMP+ $(n=12) \quad$ stage II; $n=4$, stage III; $n=3$, stage IV; $n=5$

CY-/LAMP- $(n=31) \quad$ stage IA; $n=19$, stage IB; $n=6$, stage II; $n=4$, stage III; $n=2$ 